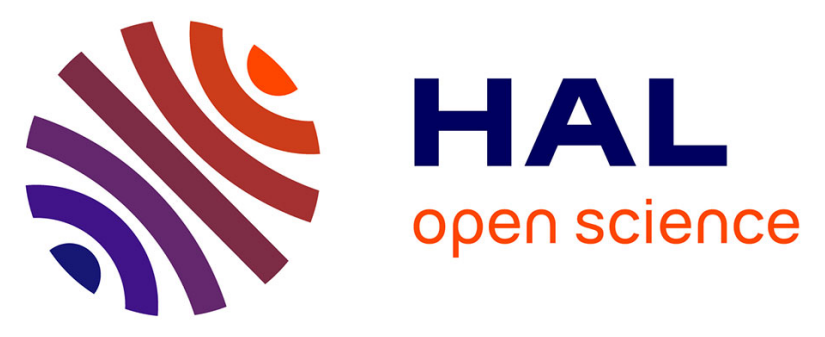

\title{
Relations sociales et solidarités collectives dans les déplacements périurbains : vers une identité de mouvement ?
}

Benjamin Pradel, Laurent Cailly, Marie-Christine Fourny, Sonia Chardonnel, Rodolphe Dodier, Sophie Louargant

\section{To cite this version:}

Benjamin Pradel, Laurent Cailly, Marie-Christine Fourny, Sonia Chardonnel, Rodolphe Dodier, et al.. Relations sociales et solidarités collectives dans les déplacements périurbains : vers une identité de mouvement ?. RTS - Recherche Transports Sécurité, 2014, Les sens des circulations, 2014 (02-03), pp.125-141. 10.4074/S0761898014002052 . hal-01670617

\section{HAL Id: hal-01670617 https://hal.science/hal-01670617}

Submitted on 21 Dec 2017

HAL is a multi-disciplinary open access archive for the deposit and dissemination of scientific research documents, whether they are published or not. The documents may come from teaching and research institutions in France or abroad, or from public or private research centers.
L'archive ouverte pluridisciplinaire HAL, est destinée au dépôt et à la diffusion de documents scientifiques de niveau recherche, publiés ou non, émanant des établissements d'enseignement et de recherche français ou étrangers, des laboratoires publics ou privés. 


\title{
Relations sociales et solidarités collectives dans les déplacements périurbains : vers une identité de mouvement?
}

\author{
Social relationships and collective solidarity in periurban journeys: \\ towards an identity of movement
}

\author{
Benjamin Pradel ·Laurent Cailly ·Marie-Christine Fourny ·Sonia Chardonnel ·Rodolphe Dodier · Sophie Louargant
}

Reçu le 25 novembre 2013 ; accepté le 29 avril 2014

(C) IFSTTAR et Éditions NecPlus 2014

Résumé Quelles sont les relations interindividuelles en jeu dans la mobilité périurbaine et que produisent-elles? Fabriquent-elles des solidarités collectives ? Définissentelles des modalités de déplacements particulières ? Tel est le questionnement et l'objet de cette contribution. Partant d'une analyse qualitative des espaces-temps de la mobilité dans le moyen-Grésivaudan (Grenoble, France), auprès d'habitants recourant aux transports en commun ou au covoiturage, nous montrons que les trajets domiciletravail jouent un double rôle de sas et de lien entre

\footnotetext{
Benjamin Pradel $(\square)$

Ecole des Ponts ParisTech, UMR LVMT

6-8 avenue Blaise Pascal, F-77455 Marne-la-Vallée

e-mail : benjamin.pradel@enpc.fr

Laurent Cailly $(\bowtie)$

Université François Rabelais, UMR CITERES 7324,

33 allée Ferdinand de Lesseps, 37200 Tours

e-mail : laurent.cailly@univ-tours.fr

Marie-Christine Fourny $(\triangle)$

Univ. Grenoble Alpes, UMR PACTE,

F-38000 Grenoble, France

e-mail : marie-christine.fourny@ujf-grenoble.fr

Sonia Chardonnel $(\varangle)$

CNRS, Univ. Grenoble Alpes, UMR PACTE,

F-38000 Grenoble, France

e-mail : sonia.chardonnel@ujf-grenoble.fr

Rodolphe Dodier $(\bowtie)$

Aix-Marseille Université, UMR TELEMME, MMSH

5, rue du Château de l'Horloge, 13094 Aix-en-Provence

e-mail : rodolphe.dodier@univ-amu.fr

Sophie Louargant $(\square)$

CNRS, Univ. Grenoble Alpes, UMR PACTE,

F-38000 Grenoble, France

e-mail : sophie.louargant@iut2.upmf-grenoble.fr
}

les sphères du quotidien parce qu'ils sont investis d'une diversité d'usages et d'interactions sociales. Le partage d'un même habitacle, de normes et de règles d'interactions mais aussi de solidarités collectives ancrées tout autant dans les territorialités habituelles (résidence, travail) que dans l'expérience de mobilité nous amène à définir une identité de mouvement. Cette dernière qualifie un sentiment de reconnaissance et d'appartenance fondé sur le partage des mêmes conditions matérielles et idéelles de déplacement, au cœur de la compréhension de l'organisation individuelle et collective des mobilités.

Mots clés mobilité · sociabilité · périurbain · identité · TIC

\begin{abstract}
What are the interindividual relationships involved in periurban journeys and what is their outcome? Do they create a sense of collective solidarity? Do they concern particular types of journey? These are the issues raised in this paper. Based on a qualitative analysis of space and time in relation to journeys taken by inhabitants using public transport or car-sharing within the Grenoble district (France), we show that journeys between home and work play a dual role of buffer and link between the spheres of daily life, because they cover a range of uses and social interactions. The notion of sharing not only a passenger compartment and standards and rules of interaction but also a sense of collective solidarity, rooted as much in everyday space (home, workplace) as in the experience of the journey itself, leads us to define an identity of movement. This includes a feeling of recognition and belonging based on sharing the same material conditions and ideals of transport, central to the understanding of the individual and collective organization of journeys.
\end{abstract}

Keywords mobility, sociability, periurban areas, identity, ICT 


\section{Introduction}

Alors que les solidarités et les mobilisations collectives associées aux navettes longue distance ont suscité plusieurs travaux [1,2], les recherches portant sur la mobilité périurbaine ont encore peu exploré les dimensions collectives et interindividuelles des pratiques de mobilité, à l'exception des solidarités internes à la famille liées à l'accompagnement des enfants [3,4,5]. L'hégémonie de l'automobile - et principalement de l'auto-solo explique en grande partie la focalisation du regard des chercheurs sur la forme « individualisée » des déplacements périurbains et sur les effets structurants de la motorisation en matière d'habiter $[6,7,8,9]$. Si ces travaux proposent des interprétations nuancées de la mobilité périurbaine, il faut reconnaître que le primat de l'automobile est au cœur des débats - et souvent des représentations négatives et caricaturales - dont le périurbain est l'objet. Dans bien des discours, la « voiture » incarne l'individualisme imputé aux périphéries urbaines et constitue une source d'aliénation individuelle et/ou de dé-liaison du social [10].

Dans cet article, nous prenons une tout autre posture et faisons l'hypothèse que la mobilité périurbaine met en jeu des relations interindividuelles et des solidarités collectives qui, sans être foncièrement nouvelles, prennent une place croissante dans le quotidien périurbain. En effet, des travaux récents $[11,12,13]$ livrent plusieurs indices de l'amplification du recours aux transports en commun (train, bus) ou aux formes d'arrangements collectifs pour gérer la mobilité : covoiturage, solidarités familiales ou amicales. L'augmentation du coût de la mobilité, la sensibilité croissante aux questions environnementales ou encore l'amélioration de l'offre de transport en zone périurbaine créent les conditions d'une inflexion timide mais réelle des comportements, sur les territoires que nous observons ${ }^{1}$. Ce recours à des formes de mobilité impliquant des solidarités collectives nous amène à interroger de manière inédite les relations sociales qui se tissent dans la mobilité périurbaine, à étudier leur rôle dans la manière de vivre et d'habiter les déplacements et, plus largement, à examiner la place des sociabilités dans la constitution d'une identité périurbaine structurée par le mouvement.

\footnotetext{
${ }^{1}$ Les enquêtes sur les alternatives modales à la voiture-solo dans les espaces périurbains français sont encore balbutiantes et fragmentaires. Par conséquent, dans cet article, il s'agit moins de tenir un discours de généralité sur la transformation des comportements de mobilité en périurbain (ce qui nécessiterait de mobiliser des données quantitatives, à l'échelle nationale) que d'analyser les implications socio-spatiales $\mathrm{du}$ recours à des solutions collectives (transports en commun et covoiturage) qui, selon plusieurs auteurs, semblent se renforcer, à partir d'un espace d'étude (le Grésivaudan) où cette évolution vers des solutions collectives est statistiquement avérée.
}

Nous entendons par sociabilité «l'ensemble des relations qu'un individu [...] entretient avec d'autres et les formes que prennent ces relations » [14]. À la suite de Gurvitch [15], nous considérons la sociabilité comme un phénomène social total engageant des rapports sociaux plus ou moins profonds, dont les figures varient selon des degrés d'intensité et de structuration, des plus organisées aux plus fluides [16]. Cette perspective sociologique nous amène à envisager la mobilité comme le lieu de la manifestation, de l'actualisation ou encore de la construction d'un large spectre de relations interindividuelles. Celles-ci renvoient aux interactions sociales engagées en situation de déplacement (lieux de la mobilité et habitacles) et aux réseaux sociaux activés dans le déplacement. Ces relations construites ou (ré)activées autour de l'enjeu de la mobilité varient selon l'engagement des individus, prennent des formes différentes suivant les situations spatiales et temporelles, mais impliquent toutes un processus de socialisation [17]. Les plus consistantes d'entre elles peuvent conduire à des identités collectives. En effet, dans l'interaction s'activent et se coproduisent des représentations, des pratiques, des valeurs, des appartenances dont l'identification et l'intériorisation sont ici facilitées par le partage d'une même situation de mobilité et d'un certain nombre de traits communs entre les individus : des déplacements domicile-travail d'actifs occupés, résidents sur un territoire circonscrit, empruntant des trajets similaires selon des horaires relativement stables. Les relations sociales engagées dans et par le mouvement généreraient alors, autant qu'elles révéleraient, des identités collectives relatives à la condition périurbaine, en participant de l'identification de l'individu à un groupe en relation avec un territoire (le périurbain) et une territorialité.

Ceci nous conduit à développer l'hypothèse d'une « identité de mouvement ». Elle repose sur l'idée que la mobilité périurbaine, pour ceux qui recourent à des modes partagés ou collectifs, suscite une forme "d'auto-compréhension » des individus [18], c'est-à-dire une subjectivité située qui se cristallise, se consolide et se manifeste à travers le partage d'une expérience pratique et d'un sens collectif $\mathrm{du}$ déplacement. Un tel processus est à mettre en rapport avec la constitution progressive et interactive d'un groupe partageant un certain nombre de similitudes qui sont à la fois générées (règles implicites de comportements dans l'habitacle) et révélées (horaires de travail commun) dans et par le mouvement. Ces similitudes objectives et subjectives sont notamment éprouvées, ressenties ou perçues dans les relations sociales, interactions et solidarités, impliquées dans la mobilité. Elles participent d'une construction et prise de conscience identitaire par un collectif autour de la mobilité comme situation et enjeu. Ainsi, pour les groupes sociaux qui recourent à des arrangements collectifs, l'épreuve et le sens partagés de la mobilité facilitent 
l'auto-identification du groupe. En un sens, cette identité de mouvement, par son caractère situé et contingent, diffère de la notion d' "identité de déplacement $»^{2}$ qui renvoie plus globalement au rôle de la mobilité (trajets, modes, lieux de destination) dans la construction de l'identité socio-spatiale de l'individu [19]. En même temps, l'identité de mouvement dialogue avec l'identité de déplacement - au sens où la conscience commune, le «nous »-qui émerge de l'expérience conjointe des situations de déplacement, repose sur, et se nourrit, d'une identité socio-spatiale plus profonde fondée sur des similitudes matérielles et idéelles en matière d'habiter. Dès lors, l'analyse des sociabilités engagées dans la mobilité périurbaine nous amène à nous poser la question de l'articulation entre deux formes de construction identitaire généralement traitées séparément dans la littérature : l'une subjective et située, en « acte » dans le temps routinier du déplacement ; l'autre structurelle et objectivée, ancrée dans l'épaisseur biographique de l'habiter.

Ces questionnements autour de l'identité de mouvement s'inscrivent dans une réflexion plus générale sur les liens entre mobilité, territorialité et habitabilité, menée au sein du programme de recherche «TerrHab ${ }^{3}$. Dans cette recherche, nous testons l'hypothèse selon laquelle la mobilité quotidienne reconfigure le rapport des individus à l'espace et fait naître une nouvelle territorialité - la territorialité mobile - dans laquelle le mouvement est un opérateur de continuité spatiale, sociale et temporelle entre les différents espaces de vie de l'individu [20]. Dans cette perspective, la mobilité ne s'oppose pas aux processus d'appropriation spatiale ni aux logiques d'ancrage mais, au contraire, y participe et se trouve de facto au centre de l'habiter et de l'habitabilité périurbaine. En contrepoint des analyses insistant sur l'éclatement et la dissociation des territoires de vie par la mobilité [21,6,8], nous appréhendons la mobilité comme un facteur d'articulation et d'interpénétration entre les territoires de l'individu. Il s'agit alors d'examiner la manière dont les sociabilités impliquées

\footnotetext{
${ }^{2}$ L'identité de déplacement souligne que la mobilité construit l'identité personnelle. Elle postule une mobilité quotidienne subordonnée à l'identité sociale de l'individu, que ce soit en termes de modes de déplacements utilisés ou de lieux fréquentés. L'identité de déplacement relève ainsi d'un ensemble de pratiques et de représentations relatives à la mobilité et qui, mettant en jeu de la continuité et de la distinction, permet le « replacement » de l'individu dans la négociation de sa position sociale, cognitive et géographique. « La mobilité serait donc ancrage tout autant que mouvement et par là même, révélatrice d'identité en fonction des modalités de cet ancrage » [19].

${ }^{3}$ Les travaux et analyses relatés ici sont issus d'un programme de recherche ANR Espace et Société (2010-2014). Il s'intitule « TerrHab, de l'habitabilité à la territorialité, et retour : à propos de périurbanités, d'individus et de collectifs en interaction ». La recherche, dirigée par Martin Vanier, avec Marie-Christine Fourny et Romain Lajarge est portée par l'UMR Pacte (Grenoble), l'UMR Citeres (Tours), l'UMR SET (Pau), l'UMR Ausser (Paris), l'EA Lidilem (Grenoble 3).
}

dans la mobilité produisent des continuités territoriales (lieux), sociales (groupes) ou temporelles (activités) autour desquelles s'organisent l'habiter périurbain. Ces continuités sont notamment issues des rapports sociaux liés aux déplacements, par lesquels se réalise l'articulation des espaces du quotidien.

\section{Le contexte de l'étude : protocole d'enquête et spécificités socio-spatiales de l'échantillon}

L'enquête qualitative que nous avons menée en périphérie de Grenoble sur les déplacements domicile-travail d'habitants périurbains et dont nous présentons ici les premiers résultats révèle que les sociabilités et les solidarités collectives engagées dans la mobilité périurbaine sont loin d'être anecdotiques. La présence sur ce territoire de ménages «innovants " ${ }^{4}$ en matière de mobilité et d'une offre altermodale conséquente explique le recours important aux solutions collectives (covoiturage, bus, TER), potentiellement sources de sociabilités. Pour autant, ces sociabilités issues de la mobilité obéissent à une structuration complexe. Elles sont nourries par l'expérience quotidienne et routinière du déplacement mais sont souvent ancrées et reliées aux autres territoires de vie : celui du travail, de la résidence, voire des deux à la fois. En outre, ces sociabilités participent à l'habitabilité périurbaine : elles constituent à la fois une «activité sociale » en tant que telle (discussions, échanges) qui permet d'habiter le déplacement et un « réseau » de relations sociales qui se présente comme une ressource et qui intervient de manière déterminante dans l'organisation de la vie quotidienne et de la mobilité. Pour interroger les sociabilités engagées dans les situations de déplacement, nous avons mis en œuvre un dispositif d'enquête inédit.

La méthode consiste à articuler un entretien « embarqué » [20] avec un entretien de réactivation au domicile de l'interviewé. L'entretien embarqué est d'abord une situation d'observation d'un trajet ordinaire de l'individu depuis son lieu de départ (domicile ou travail) jusqu'à son lieu d'arrivée (domicile ou travail) durant lequel nous essayons de repérer les manières de faire, les habitudes, les routines, ainsi que les éventuelles interactions sociales avec d'autres passagers (notamment lorsque la mobilité appelle la coprésence dans un transport en commun ou

\footnotetext{
${ }^{4}$ Par opposition à des mobilités périurbaines associées traditionnellement à l'autosolisme, nous qualifions ainsi d'innovants les ménages périurbains qui, pour réaliser leurs déplacements quotidiens, recherchent des alternatives à l'usage individuel de la voiture et expérimentent diverses solutions, souvent combinées : vélo, vélo électrique, covoiturage, train, bus, auto-stop, etc.
} 
lors de covoiturage $)^{5}$. Cet entretien embarqué permet également d'échanger avec la personne enquêtée sur le choix du mode de transport, l'organisation du déplacement et les activités pratiquées pendant la mobilité. Ensuite, l'entretien de réactivation, nettement plus long, permet de contextualiser le trajet observé en le replaçant dans l'ensemble du schéma de mobilité de la personne, dans l'organisation spatiale et temporelle de la famille et dans une perspective biographique.

L'échantillon des personnes interrogées est composé de 7 femmes et de 8 hommes, âgés de 24 à 55 ans, résidant dans la vallée du Grésivaudan ou sur ses versants et travaillant à Grenoble. Il a été composé suivant deux variables : une localisation résidentielle impliquant un temps de déplacement vers Grenoble supérieur à 30 minutes et le type de transport. Sur ce dernier aspect, nous avions surtout l'exigence d'atteindre un large panel de modes (véhicule personnel seul, véhicule personnel partagé, covoiturage formel, bus express régional, bus métropolitain, TER, vélo) et de combinaisons multimodales. Le groupe enquêté se compose de cadres et professions intellectuelles supérieures mélangeant secteur public, privé et professions libérales (8), d'employés (4), de professions intermédiaires (2) et d'un étudiant. On notera que cet échantillon est le premier d'une enquête devant compter à terme 45 personnes dans trois villes intermédiaires françaises (Grenoble, Aix-en-Provence et Tours), et qu'il a été complété par 3 entretiens exploratoires effectués en amont de cette recherche, toujours autour de Grenoble.

\footnotetext{
${ }^{5}$ Proche de la technique du parcours commenté, l'entretien embarqué s'en distingue sur plusieurs points. Il s'inscrit d'abord dans un protocole d'enquête plus large comprenant un entretien de réactivation et de contextualisation qui n'est pas un simple « retour d'expérience » [22]. Ensuite, il se déroule lors d'un trajet quotidien, dans les conditions du quotidien. L'enquêté ne peut pas prendre «plus de temps » qu'à l'ordinaire et l'entretien se termine à destination, à l'inverse des parcours en gare expérimentés par Levy [23]. On ne demande pas non plus à la personne « de recréer un parcours qu'elle a l'habitude de faire » [22], ni de « créer un parcours qui rassemble tous les endroits qu'elle apprécie particulièrement » [24] et encore moins d'être guidée par le chercheur sur un itinéraire qui lui est inconnu [25]. L'entretien se déroule dans les « conditions du réel » quitte à ce que ces dernières perturbent ou ne permettent pas de terminer la grille d'entretien. L'entretien embarqué ne consiste pas non plus à recueillir seulement du discours sur le parcours effectué. La grille d'entretien aborde des thématiques qui ne sont pas directement liées au trajet, comme l'organisation des déplacements familiaux. À ce titre, il n'est pas seulement une « mise en récit en temps réel du parcours » [22]. L'entretien embarqué repose sur le croisement interactif d'un entretien semi-directif réalisé dans l'habitacle et d'observations focalisées et flottantes. Il nécessite ainsi une grille d'entretien souple regroupant les thématiques importantes mais dont l'utilisation peut varier selon les situations rencontrées dans le déplacement, et une grille d'observation qui, en lien avec la grille d'entretien, resitue le contexte évolutif de l'entretien et permet d'alimenter ce dernier de manière dynamique. Il n'y a pas ici de caméra, ni de photographe [22].
}

Le territoire périurbain concerné correspond au « Pays du Grésivaudan » en Isère, situé entre deux agglomérations, Grenoble et Chambéry. Selon le recensement général de la population de 2010, il compte 97705 habitants et sa croissance de population s'est élevée à $14 \%$ depuis 1999 . Cet espace est caractérisé par un habitat pavillonnaire et une forte mobilité quotidienne - en distance parcourue - des habitants. Sa configuration linéaire, à l'image d'un corridor, associe toutefois une vocation résidentielle avec une vocation technologique, notamment dans les pôles d'activités proches des échangeurs d'autoroute ou de l'agglomération-centre. Le tissu économique « technopolitain » représente $19 \%$ des établissements de haute technologie de l'agglomération. De ce fait, $53 \%$ des actifs $\mathrm{du}$ territoire travaillent à l'extérieur, mais un quart des actifs sont occupés dans les polarités secondaires du territoire étudié (Crolles, Montbonnot, Pontcharra). Par ailleurs, selon l'Enquête Ménages-Déplacements de 2010, les échanges entre Grenoble et le Grésivaudan représentent $74 \%$ des déplacements automobiles quotidiens.

Cette région périurbaine est marquée par la surreprésentation des cadres et professions intellectuelles supérieures. Il accueille des familles aux revenus élevés (catégories moyennes supérieures), ce que traduit un indicateur de fiscalité pour 2006 très supérieur à la moyenne régionale et nationale. Il n'en est pas toutefois un lieu de résidence exclusif. Il se diversifie par la présence de professions intermédiaires (plus de $20 \%$ des actifs), mais également d'employés, et, dans une moindre proportion, d'ouvriers. Le gain migratoire a concerné toutes les catégories professionnelles de 1990 à 2010, tout en restant plus élevé pour les cadres supérieurs et les professions intermédiaires : plus de $43 \%$ des cadres se sont installés entre 1990 et 2000. En outre, c'est un territoire plutôt jeune, $28 \%$ de la population ayant moins de 20 ans.

Du point de vue de la mobilité, en 2010, un habitant du Grésivaudan effectue 3,5 déplacements par jour, d'une moyenne de 59 minutes, ce qui le place très légèrement au-dessus de la moyenne nationale des communes polarisées des aires supérieures à 100000 habitants $(3,11$ déplacements/jour d'une moyenne de 55 minutes). Sur ce territoire, la part modale de la voiture $(69,5 \%)$ reste prédominante comparativement à l'agglomération grenobloise ( $46 \%$ pour la Métro), mais se situe nettement en dessous de la moyenne des espaces périurbains français $(78 \%)$ puisque la voiture particulière a enregistré une baisse de 6 points entre 2002 et 2010 au profit des transports en commun (Source : Enquête ménages déplacements, 2010). Ces éléments accréditent l'idée d'un changement tendanciel des comportements de mobilité au sein des territoires périurbains lorsque ceux-ci sont bien dotés en transports collectifs comme c'est le cas du territoire d'enquête. Celui-ci est doté de quatre gares ferroviaires qui accueillent 1400 voyageurs par jour et les 
transports par car régionaux affichent sur l'ensemble des réseaux, 5000 voyageurs par jour. Ces alternatives modales et le recul de la part de l'automobile justifient dans notre enquête la focalisation du regard sur les actifs périurbains recourant aux solutions collectives (bus, train, covoiturage) pour réaliser leurs déplacements domicile-travail.

\section{Sociabilité en interaction dans les déplacements}

Les sociabilités font partie intégrante des pratiques individuelles d'appropriation de l'espace-temps du déplacement. Au même titre que les activités individuées (travail, lecture), elles participent de l'habitabilité de la mobilité, en tant qu'activité en soi, ou en tant que relation interactive et continue entre individus. Elles organisent ainsi le vivre-ensemble des habitacles, des quais de gare, des stations de bus, etc. Ce n'est pas toutefois la seule échelle des lieux du déplacement qui est en jeu : plus largement ces sociabilités contribuent à rendre habitable l'espace périurbain. Nous allons présenter ici quelques formes de sociabilités observables dans les situations de déplacements avant d'étudier le rôle qu'elles exercent dans l'appropriation sociale du temps de déplacement, puis les règles collectives auxquelles elles donnent lieu. Nous discuterons si et comment, dans le cadre de mobilités partagées, ces interactions sociales routinières contribuent à former une identité collective de mouvement.

\subsection{Quelles formes de sociabilité dans le mouvement?}

\subsubsection{Le déplacement domicile-travail ou la figure du «sas »}

La focalisation de l'étude sur les sociabilités ne doit pas occulter que le déplacement est d'abord un temps de repli de l'individu sur lui-même durant lequel il s'extrait de ses sphères d'activités localisées pour se divertir ou se reposer (lecture, sieste, musique), ou bien se livre à des activités en lien avec ces sphères : préparation du travail de la matinée, coups de téléphone personnels, organisation de l'agenda familial. De la sorte, l'espace-temps de la mobilité fonctionne comme un sas spatial, social et temporel, vécu comme tel et particulièrement important dans la vie psychique de l'individu (Figure 1). La métaphore du sas illustre bien le statut ambivalent du déplacement domicile-travail. Le trajet constitue un « espace-temps tampon », un « entre-deux », c'est-à-dire un espace caractérisé par des usages et des fonctions spécifiques entre deux temps sociaux localisés. Mais, simultanément, cet espace fait lien et se trouve doté de propriétés transitionnelles qui permettent à l'individu de glisser d'une sphère d'activité à l'autre.

Le déplacement domicile-travail renvoie ainsi à la figure de l'entre-deux, lorsqu'il prend la forme d'un temps de régénération entre deux sphères marquées par des cadres imposés (le travail et le monde domestique) : malgré les contraintes objectives (horaires, fatigues), la nécessité du déplacement est là transformée pour acquérir une valeur positive, en constituant notamment un temps d'autonomie et de centration individuelle.

Il renvoie à la figure du lien, lorsque l'espace-temps est celui où s'étend et s'amorce la vie professionnelle et/ou domestique : «C'est vrai que moi, ça peut m'arriver de devoir travailler sur mon portable, en sortant du boulot. Ben, le fait d'avoir une petite tablette, ça me permet de le poser et de travailler plus correctement »(Hervé). Cette extension des activités professionnelles, et parfois domestiques, sur le temps de déplacement participe d'une stratégie d'optimisation dans la gestion du temps. Elle peut permettre, par exemple, de mieux séparer les activités. Ainsi, travailler dans le train peut accroître l'efficacité professionnelle tout en limitant le débordement de cette activité sur la vie domestique : « le soir quand je sors d'une réunion je tape peut-être tout de suite les comptes rendus [...]. Comme ça, c'est fait en rentrant et je l'envoie tout de suite » (Nicole).

On soulignera ici que les figures du lien et du sas ne présagent pas d'une séquence unique de déplacement entre le domicile et le travail. Des activités liées à ces deux sphères peuvent s'y inscrire renforçant du même coup l'interpénétration ou la séparation dans la mobilité des logiques domestiques ou professionnelles. À l'aller, le travailleur peut passer prendre un collègue pour se rendre à un rendez-vous professionnel avant d'atteindre son lieu de travail fixe. Au retour, il en profite pour faire des courses ou passer prendre ses enfants à la sortie de l'école. On retiendra de ce qui précède que le déplacement domicile-travail doit d'abord être entrevu comme « un espace-temps » qui fait lien et tisse de la continuité entre les sphères d'activité de l'individu.

\subsubsection{L'individu mobile, entre repli et ouverture à l'autre}

Si l'expérience de mobilité est fortement "individuée », elle laisse libre cours à des sociabilités diverses qui participent aux logiques de «sas ». Dans les transports en commun (TER, bus), l'ouverture aux autres voyageurs relève davantage d'un potentiel que d'une nécessité qui s'imposerait à l'individu. Celui-ci dispose de marges de manœuvre et choisit le moment où il souhaite interagir avec d'autres. Des règles partagées, propres à ces situations de mobilité, régulent les interactions sociales : elles garantissent le respect de l'intimité ainsi que le 


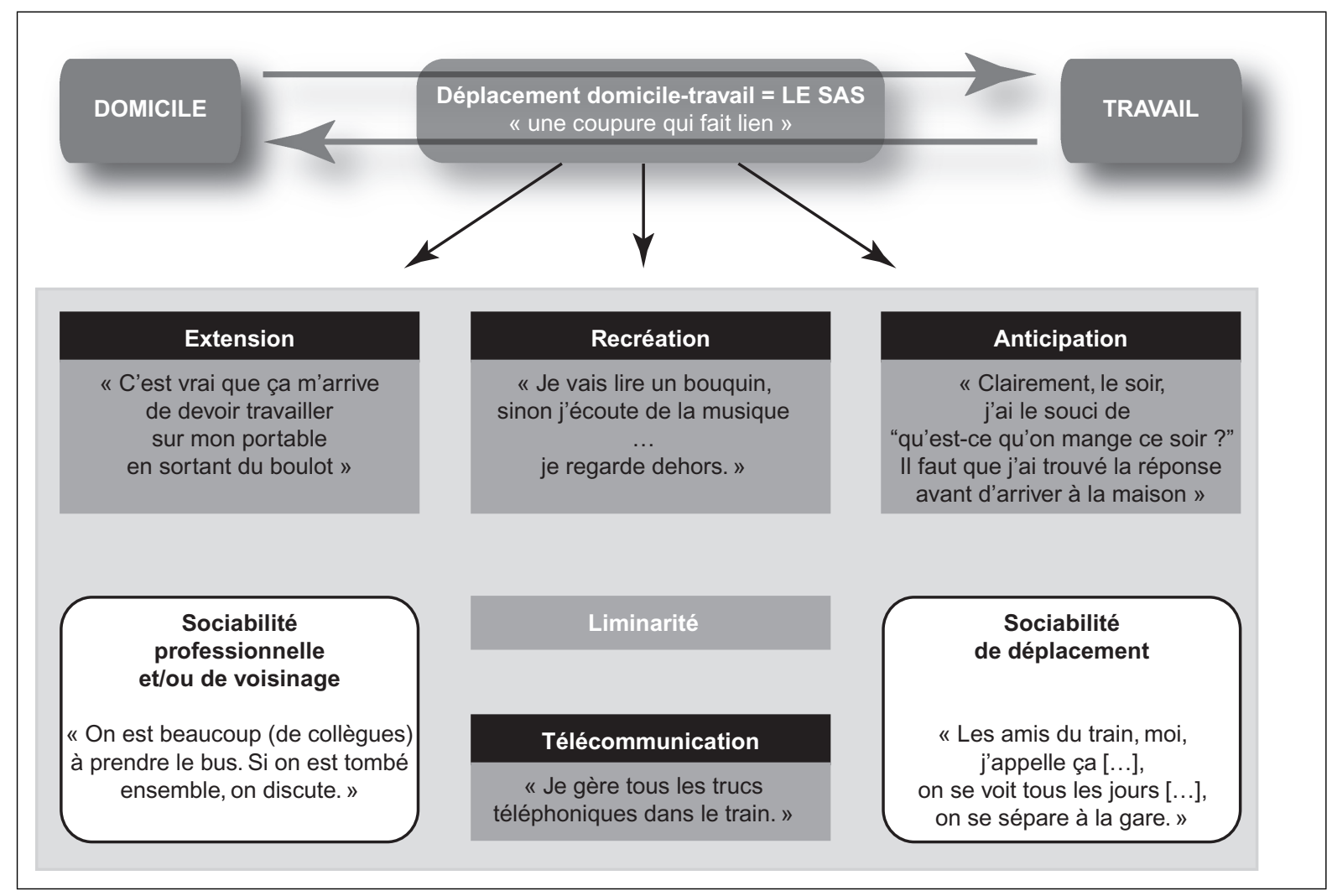

Fig. 1 Sociabilités et formes de la continuité périurbaine

principe d'engagement volontaire. Ainsi, chacun respecte la tranquillité des autres voyageurs, notamment le matin, dans une forme de compréhension mutuelle du besoin de se reposer ou des obligations professionnelles qui pèsent sur certains : «quand il n'y a rien à faire c'est plus sympa [de discuter] mais si on commence à bosser... Parfois, c'est pas pratique, mais souvent, les gens vous le disent quand ils ont un truc à faire » (Claude). On observe ainsi le respect d'une distance qui varie selon le moment de la journée ou suivant une temporalité propre à chacun. Pour cette enquête, « Je suis plutôt du matin, je suis assez réveillée, je peux être sociable. Par contre, le soir, j'ai plutôt envie d'être tranquille » (Aurélie) tandis que pour cet autre le trajet se fait « généralement seul. Parfois je rencontre des gens que j'ai rencontrés à la gare ou pendant le trajet. Quelquefois on discute mais plutôt le soir, le matin ça bosse » (Claude). Il semble toutefois que le trajet du retour soit plus propice aux sociabilités que le temps du matin davantage caractérisé par un repli de l'individu sur lui-même.

À la différence des transports en commun, la voiture partagée (covoiturage, stop) laisse davantage de place aux échanges interindividuels. L'engagement dans une même situation d'embarquement, la proximité dans l'habitacle et le caractère «personnel » de celui-ci rendent inévitables les interactions entre le conducteur et le(s) passager(s), ne serait-ce que d'un point de vue pratique, la situation de mobilité impliquant des conversations autour des conditions de déplacement, ou de réglage de l'itinéraire et des horaires. Mais, plus fondamentalement, l'échange entre les passagers fait partie de la justification sociale d'une pratique conçue d'emblée comme collective, aux côtés des raisons économiques ou environnementales. Ainsi, la recherche de relations sociales fait partie des motifs exprimés par certains : «Je préfère [être] accompagné, c'est quand même plus sympa [...] on peut discuter » (Isabelle), ou encore le covoiturage «c'est un moyen, euh pff, comment je vais dire ça, c'est un moyen assez sympa déjà de... de connaître son prochain qui habite sur le plateau [des Petites Roches dans la vallée du Grésivaudan] [...]. Donc un moyen de se connaître déjà » (Simon). Inversement, le refus de subir une relation sociale répétée peut conduire certaines personnes à ne pas choisir ou à abandonner le covoiturage au profit d'un autre mode où le rapport à l'autre est moins « obligé ». Si covoiturer ou prendre des auto-stoppeurs implique une relation sociale plus soutenue que dans les transports en commun, ces modes n'imposent pas pour autant « d'être obligé de parler tout le temps ». Là encore, un contrat implicite permet de s'entendre sur la place que l'on entend donner à la parole comme l'énonce cette personne qui prend parfois des auto-stoppeurs : « des fois j'ai envie de discuter 


\begin{tabular}{|c|c|c|c|c|}
\hline $\begin{array}{l}\text { Types de sociabilité } \\
\text { dans l'habitacle }\end{array}$ & $\begin{array}{l}\text { Degré } \\
\text { d'altérité }\end{array}$ & Intensité & Logique socio-spatiale & Modes de transports \\
\hline 1. Aucune & Nul & Nulle & $\begin{array}{l}\text { Repli sur soi, activités } \\
\text { personnelles }\end{array}$ & Tous modes sauf covoiturage \\
\hline 2. Couple, famille & Faible & Très forte & $\begin{array}{l}\text { Extension du territoire } \\
\text { domestique }\end{array}$ & Auto-partagée, principalement \\
\hline $\begin{array}{l}\text { 3. Collègues, voisins, } \\
\text { gens du village }\end{array}$ & Faible ou moyen & Variable & $\begin{array}{l}\text { Extension du territoire } \\
\text { professionnel ou résidentiel } \\
\text { hybridation des sphères }\end{array}$ & Tous modes sauf voiture solo \\
\hline 4. « Amis du train $»$ & Faible ou moyen & Variable & $\begin{array}{l}\text { Constitution d'un territoire de la } \\
\text { mobilité : sociabilités répétées, } \\
\text { déconnectées des deux bouts de } \\
\text { la chaîne }\end{array}$ & Bus, train, covoiturage \\
\hline $\begin{array}{l}\text { 5. « Têtes connues », } \\
\text { Jeunes, Chauffeur }\end{array}$ & Fort & Faible & $\begin{array}{l}\text { Constitution d'un territoire de la } \\
\text { mobilité : sociabilités répétées, } \\
\text { brèves mais faibles }\end{array}$ & Bus, train, covoiturage \\
\hline
\end{tabular}

avec eux et c'est sympa, mais y'a des moments ou je prends des auto-stoppeurs pour dépanner mais je n'ai pas forcément envie de parler au matin, et donc moi je leur fais comprendre voilà. Ce sera un trajet silencieux » (Annick).

En définitive, les situations de mobilité observées livrent des potentiels de sociabilité différents suivant les modes de transport mais mettent toujours en jeu la relation de l'individu aux autres, ce qui nous amène à questionner les types de relations sociales qui se tissent dans la mobilité.

\subsubsection{Formes de sociabilité en déplacement}

Les situations de mobilité engagent un large spectre de sociabilités qui varient selon le degré d'altérité des personnes impliquées et suivant que la relation sociale existe ou non en dehors du seul trajet domicile-travail. Le tableau 1 propose une classification des sociabilités apparues dans le discours des enquêtés, des liens «forts » aux « liens faibles ».

Parmi ces sociabilités, on peut distinguer tendanciellement cinq grandes catégories.

La mobilité est d'abord le lieu de la centration sur soi (type 1) ou sur la famille (le couple qui partage un trajet quotidien, type 2). C'est également l'espace d'extension des sphères de socialisation habituelles, situées en « bout de chaîne » (type 3). Les sociabilités professionnelles (« les collègues de travail ») sont les premières mobilisées pour l'organisation du covoiturage mais aussi dans le train et le bus où elles peuvent être fortuites ou recherchées. Il peut s'agir également des relations liées au voisinage immédiat ou plus éloigné (« les gens du village ») : dans ce cas, les sociabilités construites sur une base résidentielle trouvent un prolongement dans la mobilité. Parfois, les relations dans la mobilité conjuguent les deux types d'ancrages impliquant des collègues qui habitent dans un même secteur résidentiel ou sur un même axe de circulation (ligne de bus, trajet routier). La mobilité constitue alors un moment d'actualisation important, parfois central, d'une relation sociale dont la spatialité est plus large.

La mobilité peut également constituer un espace-temps où émerge une sociabilité autonome née de l'expérience commune du déplacement, du côtoiement quotidien et routinier des mêmes personnes, du partage des mêmes conditions et des mêmes lieux de la mobilité (quai, arrêt de bus, train, etc. type 4). Ainsi, certains font des rencontres dans le TER : « avec des amis, des amis du village, et puis d'autres gens. Les "amis du train" moi j'appelle ça. On se voit tous les jours. Oui, on se voit vraiment tous les jours. Sur le quai oui. On se sépare à la gare. » (Hervé). Les « amis » ou les « copains » du train désignent alors un groupe d'individus qui a noué progressivement des relations 
de cordialité, puis de convivialité et de sympathie à partir de la seule expérience de mobilité. Elle produit un territoire « social » qui commence et se termine avec le trajet. Le covoiturage, lorsqu'il réunit au départ des individus qui se méconnaissent, peut produire le même type de sociabilité.

Enfin, une dernière forme de sociabilité, elle aussi propre à la situation de déplacement, engage des interactions brèves et de faible intensité avec les " têtes connues », des personnes partageant le même bus ou le même train et rencontrés chaque jour (type 5). « À l'arrêt de bus à $8 \mathrm{~h} \mathrm{32}$, le matin, on rencontre un peu toujours les mêmes personnes donc on finit par un peu se connaître, on se salue aussi généralement, on se dit bonjour [...] on pourrait dire on se fait un signe de la tête quoi, autre chose, pour se dire bonjour » (Antoine). Ces échanges participent à faire de l'espace-temps de la mobilité un espace familier, avec ses repères, ses habitudes. La relation avec le chauffeur de bus, dont les enquêtés font souvent mention lors des déplacements ayant recours à ce mode de transport, peut entrer dans cette catégorie. C'est une forme de socialisation quotidienne dans la mobilité faite d'interactions brèves, souvent apaisées, parfois conflictuelles. Ainsi Sylvain nous explique qu'il est de bon ton de faire un petit signe de la main en guise de remerciement au chauffeur de bus.

Ces quelques types de sociabilité réduisent nécessairement la complexité des relations sociales : il arrive que les « amis du train » soient aussi des collègues ou des voisins. Réciproquement, les «gens du village » désignent parfois des personnes que l'on rencontre rarement au lieu de résidence mais très régulièrement dans le train. Globalement, les entretiens révèlent la diversité des formes de sociabilités en jeu dans les déplacements mais aussi leur capacité à s'hybrider ou à muter vers d'autres formes. Par le covoiturage, les collègues de travail redéfinissent leurs relations autour d'autres activités : « Le collègue, il est membre d'une association qui organise des repas, ou des soirées contes donc, moi du coup, en discutant comme ça, j'ai adhéré à cette association, et j'ai participé aussi à certaines manifestations »(Anne). Les individus diffèrent aussi par l'intensité et les formes de sociabilité qu'ils mobilisent dans le déplacement. Certains sont plus dans une logique de repli, d'autres dans l'actualisation stable et durable de relations préexistantes ou constituées dans la durée, d'autres sont plus ouverts à l'inconnu et aux relations fortuites. Un même individu peut également mobiliser différents types de sociabilité dans ses déplacements, suivant le segment (tronçon partagé, puis seul), suivant la temporalité (matin vs soir), suivant ses humeurs et ses contraintes (envie/pas envie ; travail/pas de travail). Cette variabilité des comportements de socialisation et des sociabilités à l'œuvre dans le déplacement révèle l'existence de différentes modalités dans la construction des identités individuelles de déplacement. Mais elles s'élaborent selon un ensemble de ressources et de contraintes partagées qui dessinent les contours d'une identité collective plus large.

\section{2. À quoi sert la sociabilité dans la mobilité ?}

La sociabilité n'est pas une simple interaction verbale. Elle est reliée à des activités diversifiées qui renvoient à la pluralité des manières d'occuper le temps de déplacement et d'habiter ses lieux (Figure 1). La sociabilité pose, de fait, la question des fonctions sociales conférées à l'espace-temps d'un déplacement qui s'inscrit au départ dans une logique utilitaire d'accès à d'autres lieux. Cette réflexion sur l'appropriation de la mobilité par les sociabilités s'inscrit dans un contexte où la valeur sociale du temps et de l'agenda bien rempli est particulièrement forte pour les actifs qualifiés. Ce trait de fond explique probablement que l'appropriation sociale $\mathrm{du}$ temps de déplacement apparaît non seulement comme une question pertinente dans les entretiens mais aussi comme un enjeu suscitant des stratégies à travers lesquelles les individus cherchent à mieux vivre et habiter leur mobilité. À ce titre, quels sens prennent ces interactions sociales pour l'individu mobile?

\subsubsection{Des conversations pour " occuper le temps » et parfois un peu plus...}

Pour les usagers des transports en commun, l'ouverture aux autres et les interrelations participent à définir la mobilité comme un espace-temps de détente voire de re-création pour l'individu. Les relations interindividuelles prennent la forme de conversations plus ou moins longues et constituent un moyen d'occuper ce temps libre. La parole et l'échange forment ainsi une activité à part entière qui permet de donner sens à la situation de déplacement au même titre que « lire » ou « écouter de la musique »: «souvent, le matin, je lis le 20 minutes, et le soir c'est lire des livres, des BD ou écouter la musique, et discuter avec les amis » (Sylvain). La conversation peut faire du déplacement un moment convivial, voire enrichissant pour l'individu. Elle permet d'échanger sur divers sujets ou de régler des problèmes familiaux, professionnels ou de voisinage. Elle est comparée par un enquêté au «bistrot», l'analogie pouvant renvoyer à un même type de lieu de socialisation et de transition entre le travail et le domicile où se produisent des : «...débats d'idées. Mais il y en a, dans la voiture, des choses comme ça qui changent, quoi, il y a des histoires avec... qui se traitent comme ça, enfin, c'est un lieu... comme un bistrot, quoi, c'est un lieu où des choses se passent, mais là, ça se passe dans la voiture, quoi »(Simon). Quand les relations s'inscrivent dans la durée, elles s'approfondissent et donnent lieu à des conversations personnelles, parfois intimes. Pour 
les familles ou les couples, le déplacement est à la fois le prolongement du monde domestique et un espace-temps privilégié de conversation, d'échange, qui compte dans la vie du ménage. "Bien, on discute avec mon mari bien sûr. Sur euh. .. ben la vie quotidienne, les problèmes, voilà les soucis, les vacances. Ça nous permet aussi pendant ce temps-là, et bien de communiquer quoi. [...] Voilà, on fait pas le trajet seul, du coup on communique le soir quand on a eu des problèmes au travail. Du coup ça nous permet aussi et ben si on a eu des soucis, d'évacuer un peu. C'est bien d'en parler. Donc on parle et puis ça va tout de suite mieux quoi » (Claudine).

\subsubsection{La sociabilité :}

\section{l'extension du domaine de la mobilité}

Sans contradiction avec la fonction précédente de recréation, les sociabilités qui se déploient dans la mobilité impliquent souvent des groupes préconstitués (collègues, voisins, parents d'élèves) qui convertissent cet espacetemps de la mobilité en extension des sphères d'activités habituelles. Lorsqu'il implique des collègues, le déplacement est alors un temps privilégié de prolongement des relations professionnelles parfois consacrées au travail (comme régler ensemble certains dossiers), mais aussi à des conversations ordinaires qui humanisent ces relations de travail. De la même manière que certains collègues se retrouvent en gare, d'autres cherchent à partir ensemble pour prendre le bus, produisant ainsi une certaine continuité : " Je dis que moi je pars vers telle heure tout le monde dit vers là, on s'adapte, on part seul ou ensemble, on regarde pas les horaires on arrive et puis, le bus est pas là on dit tant pis, on discute avant que le bus arrive. »(Sylvain) (Photo 1). Cette socialisation professionnelle hors du lieu de travail, dans la mobilité, se retrouve également dans les pratiques de covoiturage : « quand j'ai mes collègues, c'est bien car on peut discuter, voilà, si je faisais tous les jours du covoiturage, je pense que j'allumerais pas la radio pour me tenir compagnie, donc moi je dis que c'est bien, car on peut discuter » (Marie). Le partage du temps de déplacement avec des collègues de travail représente une forme d'engagement, le matin, ou de dégagement progressif, le soir, de la sphère professionnelle. Il remodèle les relations professionnelles avec d'autres registres. "Le covoiturage ça arrive fréquemment qu'on parle travail, du coup la journée, elle commence à 7 heures du matin, dans la voiture mais après, on peut aborder des sujets plus... plus personnels. Donc, c'est. . . ça n'a jamais été pesant » (Anne). Cette extension des activités touche aussi d'autres sphères : certaines personnes se retrouvent dans le train pour échanger ou travailler sur des dossiers relatifs à la sphère associative. Dans ce cas, la mobilité constitue un moment d'actualisation d'une relation qui, pour l'essentiel, se développe dans un autre cadre, et constitue un lieu relié à d'autres lieux.

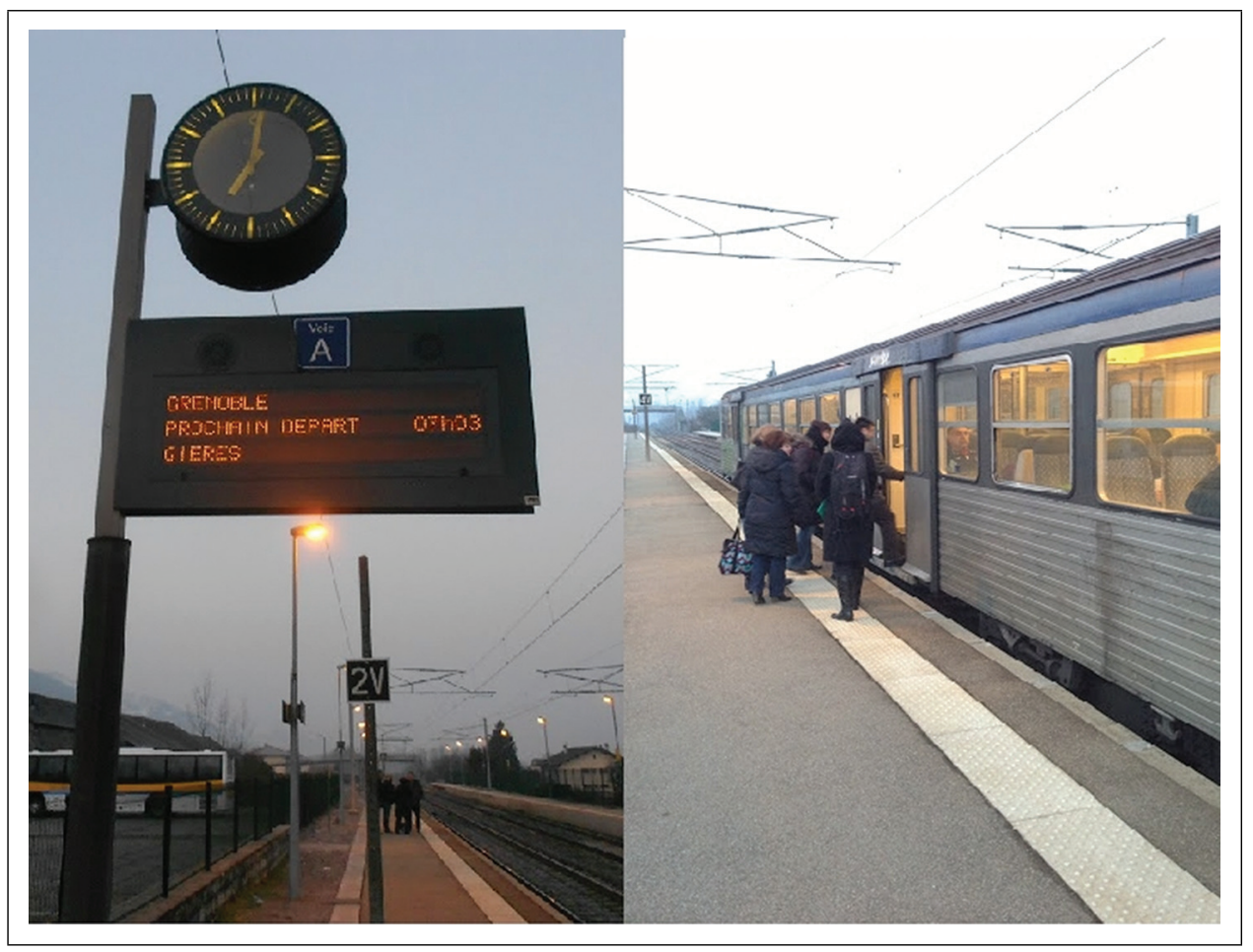

Photographie 1 : Gare de Lancey, 7 h 00 du matin, un groupe de collègues monte dans le train. Source : B. Pradel 


\subsubsection{Télé-communiquer :}

\section{le téléphone et l'internet comme « habitat-mobile»}

L'utilisation des technologies de l'information et de la communication dans les pratiques de déplacement contribue à l'habitabilité de la mobilité périurbaine. Le rapprochement des technologies débouche sur des objets numériques nomades de plus en plus hybrides (Smartphones, notebook, tablettes, liseuses) qui offrent un ensemble de fonctionnalités (jeux, Internet, téléphone, appareil photo, traitement de texte, etc.) qui participent de l'habitabilité du déplacement. D'abord, et comme il en sera question plus spécifiquement dans la troisième partie, ces objets nomades permettent d'activer des réseaux facilitant la mobilité. Les fonctions d'interface des Smartphones avec des réseaux de géolocalisation, «c'est pour mon portable qui fait GPS. » (Olivier), ou avec des réseaux d'information en temps réel, « je m'en sers pour voir si le train est bien là » (Claude), facilitent le déplacement. Ils rassurent l'individu dans sa mobilité et ont une fonction de sécurisation du trajet en tant qu'ils permettent une connexion toujours possible à des réseaux multiples engagés dans la mobilité. Cette fonction participe à faire du déplacement un temps plein, tout comme les différentes fonctions techniques qui ouvrent sur toute une gamme d'activités possibles qui remplissent le temps de trajet selon les besoins et les envies : «Je m'en sers pour écouter de la musique ou des émissions de radio en podcast. Je peux faire mon mail aussi par téléphone. » (Claude).

Les logiciels de jeu intégrés permettent de passer le temps. La fonction réceptrice permet de s'informer des actualités : « dans le train surtout, j'écoute la radio. Ça m'occupe. Et du coup je me détends, comme dans les transports. Ça permet de patienter mieux. »(Hervé). La fonction MP3 permet d'écouter de la musique «j'écoute de la musique sur le... sur le fameux Smartphone qui sert à tout !» (Olivier). La fonction communication enfin permet de passer des appels personnels : « je l'utilise dans la voiture souvent parce que j'ai pas eu le temps de rappeler quelqu'un avant »(Sébastien). En ce sens, les smartphones renforcent cette sensation de «sas » où le déplacement est un espace-temps à soi, investi par des activités pour soi qui s'insère entre les différentes activités qui remplissent la journée. D'un autre côté, ils participent de la dimension «transitionnelle » du déplacement en organisant l'engagement ou le dégagement de l'individu de ses différentes sphères d'activité entre lesquelles il chemine. L'ordinateur portable quant à lui peut transformer l'habitacle en bureau : « le temps dans le bus, je l'utilise beaucoup. J'ai mon ordinateur, je peux déjà taper des mails, je peux travailler sur des textes » (Nicole) et pour ceux qui n'ont pas d'ordinateur, l'envie est là d'utiliser le temps du train : « tu as même des prises! Pour travailler, si j'avais un ordinateur c'est super je trouve »(Hervé). Ici, l'ordinateur est utilisé en rapport avec le travail, mais sans forcément activer sa fonction de connectivité avec des réseaux. Associé à un ordinateur portable, le Smartphone transforme véritablement l'individu en travailleur mobile : « je gère tous les trucs téléphoniques dans le train puis en plus ça me sert de me connecter à l'ordi si je veux faire le mail »(Claude). Mais si jouer, s'informer, écouter de la musique, taper un texte sont des activités individus-centrées au même titre que la lecture, l'appréciation du paysage ou la sieste, la fonction émission/réception d'information en temps réel peuvent, à tout moment, rompre la logique de sas. C'est pourquoi, pour certains, le téléphone portable représente un «fil à la patte » qu'il convient parfois d'éteindre : «Non, je ne passe pas de coups de fil. Non. Non, vraiment la lecture... uniquement » (Anne). En revanche, pour d'autres ces outils nomades sont aussi utilisés en lien avec « les deux bouts de la mobilité ». Ils permettent de préparer les activités à venir, organisant ainsi la continuité de la journée en lien avec d'autres individus. Les sphères d'engagement peuvent être d'ordre familial : « là, j'appelle juste ma femme pour lui dire qu'on est en bas, en train de l'attendre » (Simon) ou amical : « après la journée de boulot, je reprends la voiture, et après en principe c'est téléphone et hop, je vais voir des amis » (Sébastien). Elles peuvent être d'ordre professionnel : « le matin c'est les trucs de boulot, si c'est le soir ça peut varier, boulot, lecture » (Claude).

Ainsi, les objets numériques nomades participent de l'habitabilité du déplacement et de l'habitacle en reliant l'individu à ses activités quotidiennes et aux protagonistes avec qui il s'y engage, mais aussi en offrant de possibles activités déconnectées remplissant le temps de trajet. C'est à travers ce potentiel de connexion et déconnexion sociale offert par les terminaux mobiles que l'individu peut organiser l'espace-temps du déplacement en tant que sas ou espace transitionnel. Autrement dit, l'individu mobile développe un mode d'habiter entre repli et ouverture à l'autre selon ses besoins et ses humeurs.

\subsection{Sociabilité, mobilité et règles collectives}

Cette capacité individuelle et collective à habiter la mobilité contribue à l'émergence d'un ensemble de règles partagées et intériorisées par les individus mobiles au fil des trajets quotidiens domicile-travail. Avec les activités, ces règles participent de l'habitabilité de l'espace-temps du déplacement. Elles régulent les interactions sociales dans les situations de coprésence. Elles relèvent d'une compétence permettant à chaque personne d'organiser son déplacement en bonne entente avec les autres dans les lieux de la mobilité. Nous prendrons ici l'exemple des trajets en bus et de ses règles tacites qui permettent de souligner la dynamique 
socio-spatiale de la socialisation dans et par la mobilité. Généralement, les passagers privilégient les places seules, posant leurs affaires sur le siège adjacent pour signaler la limite de leur territoire personnel. Ainsi, il est mal vu de se placer à côté de quelqu'un ou de lui demander de se déplacer s'il reste des places seules : " j'ai vu une dame âgée, arriver vers un jeune et lui dire : "Laisse-moi ta place". Et je trouve qu'il y a des façons de demander, et en plus il y avait des places ailleurs. Pour moi, c'est inacceptable. » (Aurélie). Ensuite, certaines places sont plus recherchées que d'autres «parce qu'on peut mettre les jambes. [...] Et souvent c'est les places où y a le plus de gens. C'est soit devant, là, tout devant, en deux ans je commence à connaître un peu les places (rire) » (Sylvain). « Connaître les places » relève d'une forme de connaissance des règles de l'habitacle, c'est-à-dire des normes collectives d'allocation des places permettant de l'habiter collectivement. Enfin, certaines règles de sécurité organisent le remplissage du bus : «le chauffeur avait refusé des passagers parce qu'il était plein, parce qu'il y a que des places assises et sur l'autoroute les gens peuvent pas être debout. » (Antoine). Le chauffeur, la figure tutélaire du bus, fait respecter la règle.

Des règles liées à la politesse émergent dans la situation de déplacement : « on se fait un signe de la tête quoi, autre chose, pour se dire bonjour » (Antoine). Les rituels de salut entre les individus mobiles renvoient au groupe assez défini des adultes actifs périurbains qui se croisent et partagent une même vision et fonction du déplacement. Ainsi, même s'ils sont collègues au travail, chacun respecte la tranquillité de l'autre : " des fois on discute, des fois chacun fait sa vie. Il n'y a pas de règle préétablie, on se connait depuis des années. C'est comme les vieux couples, quand on a plus envie de parler on ne parle plus » (Antoine). Ce respect de la tranquillité de l'autre est une règle partagée qui soutient le développement d'un sentiment d'appartenance à un groupe éphémère mais quotidien, constitué dans l'espace-temps de la mobilité. Ce dernier se construit et se révèle aussi par distinction d'avec d'autres groupes qui ne partagent pas les mêmes règles : " c'est rare qu'il y ait des personnes qui rentrent et rigolent hein. On est tous très... bah le soir on est paumé, on a travaillé toute la journée, on somnole, comme le matin quand on n'est pas encore réveillé, y'a plus d'animation dans le 6020, avec les scolaires qui rentrent» (Nicole). Le calme partagé comme marqueur du groupe s'articule avec les modalités d'appropriation de l'habitacle qui s'organisent selon un zonage bien compris de tous : « je me mets jamais à l'arrière. Ces places sont réservées aux collégiens qui aiment bien se retrouver au fond. Donc on les laisse entre eux » (Sylvain). Ici, l'utilisation du pronom personnel «on » qui distingue les actifs des scolaires, renvoie à l'auto-compréhension du groupe de travailleurs dans l'épreuve de la mobilité partagée avec un autre groupe : « les jeunes qui se mettent au fond, ils mettent la musique avec leur portable à fond là. Après bon, c'est le transport en commun on le sait hein » (Sylvain). Dans la maîtrise des codes implicites régissant l'habitacle s'opère une différenciation par l'affirmation d'une altérité entre les adolescents et les adultes renvoyant à un processus de socialisation qui participe de la construction identitaire de l'individu dans et par la mobilité. Cette répartition spatiale s'apparente à une «lutte des places » [26] dans laquelle s'affirment et se côtoient les différences alors que les espaces publics du périurbain se caractérisent par une certaine distance sociale entre les générations [27]. Ainsi, le partage de l'habitacle oblige à une négociation autour de règles communes qui participent, au final, à l'identification d'une même condition périurbaine liée au déplacement malgré les différences entre générations.

Les règles du mouvement sont l'indice de la constitution d'un groupe informel lié aux pratiques de mobilité qui donne au déplacement une épaisseur sociale révélant les premiers jalons d'une forme d'identité collective de mouvement. Intériorisées au fil du temps, ces règles sont en partie relatives au type de déplacement périurbain étudié (domicile-travail), à l'homogénéité intrinsèque des protagonistes (peu de touristes, beaucoup d'habitués) et à l'offre de transport présente dans le territoire. Ces conditions partagées facilitent l'identification interindividuelle des périurbains, l'élaboration et l'apprentissage des règles de sociabilité, notamment de la part des plus jeunes. Des règles similaires s'appliquent aux co-voitureurs avec une emphase mise sur l'importance de la conversation relative au choix même de ce mode de déplacement. Leur existence révèle un processus de construction d'une signification partagée du déplacement révélant des manières collectives d'habiter l'habitacle et, plus largement, d'habiter socialement la mobilité en tant que modalité de l'habiter périurbain. Cette épaisseur sociale de la mobilité est un révélateur de la fonction intégratrice du déplacement dans la constitution d'un système territorial.

\section{Les sociabilités et solidarités collectives comme ressource}

Un second facteur de continuité peut être mis en évidence par l'articulation entre les relations sociales localisées (sur les lieux de travail, de résidence ou d'autres activités) et les sociabilités nouées dans la mobilité. Ces interactions démontrent que si l'espace-temps du déplacement peut faire territoire, comme le montre la partie précédente, il n'est pas dissocié de la sociabilité générale de l'individu. Par exemple, le réseau d'interconnaissances de proximité est souvent activé dans la réalisation de la mobilité. Ce réseau constitue un recours essentiel dans un contexte où les 
alternatives modales sont faibles. Il garantit la possibilité de circuler en cas d'aléas, ou permet d'instaurer des formes collectives « d'auto-organisation », qui répondent à des motifs économiques ou idéologiques. De même, des personnes partageant des déplacements similaires construisent des liens d'entraide, notamment à travers le partage d'informations sur la circulation. Réciproquement, ces liens engagés autour de la mobilité conduisent à structurer des relations sur les lieux de vie et à donner ainsi de la consistance aux ancrages locaux. Ce processus révèle des liens étroits entre sociabilités de proximité et mobilité, qui nous conduisent à poser les premières comme des ressources augmentant les capacités de mobilité et palliant la faiblesse des ressources fonctionnelles. Il se manifeste aussi par la construction de collectifs organisés sur la base d'actions ayant la mobilité pour objet. Cette articulation déplacements/résidence contribue à construire et spécifier l'identité de mouvement que nous cherchons à identifier. Elle prend certes la forme d'une identité socio-spatiale, avec des groupes partageant un même espace résidentiel et structurés autour de pratiques communes. Mais celle-ci n'est finalement que le résultat de liens constitués à l'occasion des déplacements et agencés par les comportements de mobilité.

\subsection{Formes et conditions \\ de l'actualisation des ressources sociales}

La notion de ressource a l'intérêt de situer le rapport entre sociabilités et mobilité en regard d'une action. À la fois insérée «dans un processus de production » et « dans le résultat de celui-ci » [28], la ressource permet de mettre l'accent, non pas sur l'état des réseaux sociaux des individus, mais sur leur capacité à être activés et transformés dans la réalisation de la mobilité. Deux types de dynamiques se distinguent assez nettement, par la nature des réseaux sollicités, les motifs invoqués et les degrés d'engagement : d'une part la réponse à un aléa, d'autre part la mise en place d'une organisation collective pérenne.

Pour des personnes dont l'activité est tributaire du déplacement, les aléas sont bien sûrs nombreux : voiture en panne, bus raté, route barrée, indisponibilité du partenaire habituel ou incapacité momentanée. Dans ces cas, le recours à autrui et l'entraide sont la norme : " Jean-Louis, il avait une patte cassée [...] du coup, on le récupérait là et on l'emmenait, quoi ! »(Laurence). Ce recours peut aussi pallier les effets de l'éclatement des déplacements au sein de la famille. Le voisinage vient là assurer une assistance de proximité orchestrée à distance : «j'étais à Nice quand ma fille m'appelle pour me dire qu'elle avait raté son bus pour rentrer du cheval » (Jean-Pierre). Les réseaux sociaux fonctionnent en toile de fond assurantielle, pour faire face à des risques rares mais prévisibles : «j'ai des copines qui sont infirmières. .. Elles, quand elles savent que la météo va être mauvaise, elles dorment en bas, elles s'organisent entre elles et elles dorment chez des copains. » (Jean-Pierre). Ces solidarités pallient l'absence d'équipements et diminuent la vulnérabilité issue d'une situation contrainte. Elles permettent également une relative confiance sociale : l'individu périurbain n'est pas isolé et peut trouver des recours en cas de difficultés.

La solidarité peut également devenir le liant d'une organisation collective régulière. Le passage à un système d'entraide organisé existe d'abord au sein de la famille. Il prend fréquemment des formes plus extensives, soit fondées sur des relations existantes, soit en en créant de nouvelles, par exemple par l'organisation d'un réseau dédié à la mobilité (systèmes de covoiturage plus ou moins structurés). Des réseaux différents sont parfois articulés : les agencements peuvent croiser liens familiaux, connexions technologiques (internet) et localité : Martin prend ainsi sa femme sur son lieu de travail lors du retour, et prend en même temps un covoitureur «officiel " passé par le site internet dont il s'occupe et qui habite dans le même village. Ces agencements mêlent également réseaux générationnels et biens transportés : Bernard récupère les sacs de ses enfants collégiens, qui font le trajet à pied plus tôt que lui en se débarrassant de leur cartable pour plus de confort, et fait profiter du service les enfants du voisinage.

Une première condition de cette mobilité partagée est celle de l'ajustement des déplacements, tant du point de vue des horaires que des trajets. Les chaînages des déplacements, dans le couple ou entre connaissances, demandent souvent des réglages complexes. Par exemple, Pierre fait de la musique le mardi dans l'agglomération. Il y va en voiture et y retrouve une personne de son village venue également en voiture. À l'issue de la répétition, ils se suivent jusqu'au lieu de travail de Pierre, qui laisse là sa voiture, et ils remontent ensemble. Le lendemain, Pierre rejoint son travail en stop... La coordination devient en ce cas une tâche complexe. De fait, plusieurs enquêtés expliquent comment ils s'organisent avec leur conjoint pour gérer la mobilité de toute la famille : " chaque week-end, avec ma compagne, on prend l'agenda et on fait le planning de la semaine. Pour les transports domicile-travail, les réunions des uns et des autres [...] les activités extraprofessionnelles type, courses ou loisirs, qu'on va faire en semaine [...] Qui emmène qui, quand, à quelle heure. [.. .] J'ai des collègues qui ont carrément, sur leurs Smartphones, un calendrier partagé » (Simon). Le covoiturage, quant à lui, est tout entier affaire de coordination. Le système le plus pratiqué et le plus pérenne que nous avons pu observer prend la forme de mini-réseaux locaux, organisés autour d'un trajet. Les participants réunis par un parcours semblable et le souhait de covoiturer exposent leurs offres de déplacement 
et/ou s'inscrivent pour participer à des trajets. Le partage en ligne des agendas et la programmation des déplacements conditionnent ainsi des déplacements groupés. La structuration est légère, sans système d'obligation, mais elle demande une anticipation des activités : «c'est l'avant-veille qu'il faut que j'y pense, moi. Parce que le lendemain, j'ai [badminton], je reviens de là-bas et je remonte, il faut que j'y pense 3 jours à l'avance » (Jean-Pierre). Ce réseau a été mis en place par quelques personnes partageant le même trajet. L'un, ayant des compétences informatiques, a mis en place un site affichant les déplacements à partager des adhérents. Ce système a progressivement pris de l'ampleur puis s'est subdivisé en sous-groupes de cinq-six personnes. Il fonctionne comme un système coopératif, sans échange d'argent : le gain financier pour un passager est considéré comme une compensation de sa perte d'autonomie : " en gros, c'est celui qui ne prend pas sa voiture qui fait un sacrifice quelque part [...] il perd en souplesse » (Simon). Il existe néanmoins des règles tacites de don/contre-don et il est attendu de chacun qu'il assure de manière équilibrée les rôles de conducteur et de passager sous peine de mettre en danger l'informalité de l'entraide. Les pratiquants soulignent également l'importance de l'interconnaissance née ou consolidée par ce covoiturage. Les relations plus étroites entre membres d'un sous-groupe font que les mini-réseaux fonctionnent bien, alors que le système initial ouvert à tous n'avait pas perduré.

Pourquoi cette coopération ? Si les relations affectives font de la famille ou des amis proches un cadre d'entraide attendu, quels sont les facteurs déterminant un engagement plus important et permettant notamment de supporter les lourdeurs organisationnelles ? Les motifs d'ordre fonctionnel, comme faciliter le déplacement ou mettre en place une alternative au déplacement quotidien qui défaille, s'avèrent peu durables et insuffisants à surmonter les contraintes. En revanche, le motif environnemental est plus évoqué : «moi, pour me déculpabiliser, tous les stoppeurs, je les prends. [...] Pour me déculpabiliser d'être toute seule dans la voiture! J'avoue qu'hier, je suis descendue, j'en ai pris un. Il y en a pas mal, de jeunes qui font du stop sur... » (Laurence). Il peut aussi prendre des formes militantes : « Moi, c'est à $100 \%$ [par militantisme écologique]. Parce que de toute façon, moi, je ne paye pas mon essence, moi. Donc, forcément, ce n'est pas économique, c'est écologique »(Fabrice). Mais la coopération relève aussi plus simplement du compromis réalisé entre les aspirations résidentielles et les convictions écologiques : « on ne va quand même pas quitter tous ces villages pour la ville, sous prétexte qu'ils sont loin, quoi. Il faut plutôt trouver des solutions de covoiturage, de transports en commun. Il faut que les gens se... parce qu'ils sont bien à la campagne, oui, c'est indéniable » (Fabrice).
Le coût n'est guère avancé, bien qu'il soit indiqué comme une motivation « pour d'autres », ceux qu'on covoiture, plutôt que pour soi. Les caractéristiques sociologiques de notre échantillon interviennent sans nul doute : non seulement nous sommes dans un secteur socialement aisé, mais aussi dans un périurbain montagnard particulièrement attractif pour des socio-styles conjuguant sensibilités écologiques et pratiques sportives. À la différence d'autres enquêtes [29], se développe ici une solidarité d'échelle micro plus nette, en réponse à la perception d'une nécessaire solidarité environnementale à une échelle plus globale.

\subsection{Entre proximité sociale et proximité géographique, la solidarité dans le mouvement}

Si l'entraide est activée pour organiser la mobilité, il existe aussi une solidarité de condition qui s'opère avec tout individu, connu ou non, empêché dans sa mobilité. Le stop est ainsi couramment pratiqué, autant comme conducteur que comme «stoppeur ». Il demande un minimum de vigilance mais n'est pas perçu comme un risque ou un danger notable : «moi, si je vois quelqu'un qui fait du stop, s'il n'a pas une trop mauvaise tête, même des fois s'il a une mauvaise tête, je le prends quand même. Des fois, je m'en veux parce que... Alors, moi qui reviens tard le soir, à 22 h 15, des fois, je me dis : "Oh, là, là, allez, je le prends parce qu'il n'y a plus personne, après, à cette heure-là, le pauvre, il va rester...” Mais des fois, je me dis : "Mais je suis folle! Je ne devrais pas" » (Laurence). Cette solidarité envers des inconnus est cependant inscrite dans une géographie très particulière, fonction de la structure $\mathrm{du}$ réseau routier. De l'agglomération grenobloise vers le lieu de résidence, le stop est pratiqué uniquement lorsque la circulation devient locale (et pour l'un des sites d'enquête, précisément après la bifurcation avec la route nationale) lorsqu'il n'y a plus d'ambiguité sur la destination. Inversement, ne pas prendre un stoppeur à la sortie du village est décrié : «j'attends les voitures et quand elles arrivent, et ben, il y en a un qui me passe sous le nez. Il y en a encore qui font ça. Je trouve ça honteux » (Fabrice). Certes, la géographie du site enquêté favorise ce phénomène : les implantations résidentielles prennent la forme d'îlots pour lesquels le nombre d'accès est restreint. Ce comportement ne peut être totalement assimilé à une solidarité de type territorial fondée sur une interconnaissance, un sentiment d'appartenance ou une proximité sociale. En effet, on prend aussi bien les personnes en insertion " pas toujours très clean » que les touristes : il n'est pas nécessaire d' « être » de la localité. L'identité de « condition » semble ainsi s'articuler avec une proximité de réseau. 
En dehors de ce cas particulier du stop, la coopération fait intervenir à la fois une proximité physique, construite autour du lieu de travail ou de résidence, et une similitude dans le trajet. L'endroit de la dépose est ainsi important, car il détermine les possibilités d'intermodalité. La configuration du réseau est également essentielle : selon l'endroit desservi ou selon l'usage d'une autoroute, le partage est possible ou non. Les collectifs ainsi créés sont donc des collectifs structurés par des segments de réseau.

\subsection{Les effets de la solidarité de mobilité dans les liens sociaux}

Les liens d'entraide, qui activent des réseaux sociaux de proximité ou qui conduisent à des formes d'organisation collective, sont-ils susceptibles de produire des liens hors du champ de la mobilité et de contribuer ainsi à des formes de continuité dans les réseaux sociaux?

Un des premiers effets de l'entraide de mobilité consiste en la consolidation des relations de voisinage. Dans un cadre périurbain où la gestion de la distance sociale constitue une compétence essentielle [30,31], le lien constitué pendant le déplacement transforme le côtoiement en interconnaissance. Avant le covoiturage, on est voisin sans se connaître, avec le covoiturage, s'établissent de véritables relations de voisinage : « ça devient des gens qu'on est amenés à voir, à côtoyer de manière relativement suivie, quoi. Et du coup, ça ouvre quand même pas mal, quoi... c'est quand même un des moyens de connaître des gens dans le village [...] ça se traduit par des échanges de services et tout ça, [...] avec un autre gars avec qui on covoiture, prêt d'outils, ça, ça s'est fait, quoi. Apéro, café, enfin, c'est arrivé aussi, quoi. Des discussions... » (Simon). Des relations banales mais intéressantes dans la mesure où elles témoignent d'une territorialisation par le renforcement des systèmes sociaux localisés.

Le covoiturage répété permet aussi une certaine intimité car « à raison d'un face-à-face d'une heure par jour, on finit par bien se connaître » (Anne). La mobilité n'est donc pas exclusive de relations moins éphémères. Les réseaux d'interconnaissance ainsi constitués peuvent se prolonger sur d'autres activités : sortie de loisirs le soir ou le week-end. La mobilité partagée reste d'ailleurs structurante de ces partages : quand certains " se font » un cinéma ou un restaurant, d'autres en couple «se font une voiture » avec des amis (Laurence). L'expression rend compte du fait qu'un projet de loisirs dans l'agglomération est diffusé dans le réseau social, notamment auprès des covoitureurs, pour que l'opportunité de déplacement soit saisie, pour une activité commune ou non. Ces liens sont d'autant plus actifs que la mise en place des déplacements de travail conduit à une parfaite connaissance des agendas de chacun, permettant de générer des routines organisationnelles. Les aspects les mieux connus de ses partenaires de mobilité sont certainement ceux de la situation spatiale et de la temporalité de leurs activités. Ils permettent d'avoir une idée de leur localisation et ainsi des « ressources » potentiellement disponibles.

Enfin, ces solidarités de réseaux augmentent les capacités de maîtrise de sa mobilité. Téléphone, internet et smartphone aidant, les échanges d'information sur la situation du réseau routier se font en temps réel : « quand il y a un accident je pense qu'ils l'afficheraient là, ce qui pourrait tout boucher, donc après comme je connais d'autres personnes qui font le même trajet, on a l'habitude le matin quand on sait qu'on part à la même heure de s'envoyer un texto, pour dire ça roule, il y a un accident, c'est bouché. On le fait surtout quand c'est bouché, quand il y a un accident ou un problème, on s'envoie un message pour dire "ne passe pas par là" " (Isabelle). Ces informations solidarisent et confortent le réseau. Elles conduisent aussi à le configurer sur des similitudes de trajets et de temps. Des formes de sociabilité automobile procèdent de la même logique. Pour fluidifier le mouvement, on ne fait pas valoir son droit à passer et chacun prend sa part d'attente pour le bon fonctionnement de l'ensemble du trafic : «ici, c'est priorité à droite mais on passe un sur deux, sinon personne arrive chez soi à cette heure-ci » (Antoine).

De manière plus structurante, ces liens peuvent se transformer en action collective. Notre échantillon, bien que restreint, laisse entrevoir divers types d'engagements autour de la question de la mobilité. La création d'une plateforme internet gérant le covoiturage d'une association fait école : " étonnamment, ça le fait mieux que pas mal de sites. Il y a une nana, là, du coup, qui m'a contacté, qui covoiture sur un autre mini-réseau, bon, qui est assez peu actif, aussi, parce que c'est vers Meylan, ils sont beaucoup moins, ou ils ont du mal à se mettre en route, et en fait, elle va essayer de vendre ça à sa boîte, quoi. Le même type de synchronisation » (Simon). La fermeture d'une gare a entraîné un mouvement collectif : « on a mis le feu aux rails à Pontcharra, ils voulaient fermer la gare! Il y a quelques années là, on a bloqué les rails, et on a eu les CRS et tout pour obtenir que l'arrêt de Pontcharra soit maintenu, ils voulaient fermer des arrêts. Alors on a monté une assoc' qui s'appelle "Pontch'arrêt", et on s'est battu avec les élus. Et on a gagné. Mais ça a duré longtemps hein, 2 ans à peu près » (Hervé). Des prescripteurs de mobilité diffusent la bonne parole autour d'eux : « les collègues, je les ai pris par la main et j'ai dit “Tiens, on va pas prendre la voiture, on va y aller avec le car", et je vais la chercher à la maison, on y va ensemble à l'arrêt de bus. Au moins on va ensemble dans le car ou acheter ses billets etc. Et ils ont fait ça une fois et après ils voyaient ce que c'est concrètement et après ça les motive » (Nicole). 
Cette dynamique participe à diffuser des pratiques à la fois sur un objet commun de mobilité et sur une logique spatiale, aréolaire ou réticulaire, de condition et de besoin de déplacement. Encore émergente, elle peut néanmoins révéler des capacités à générer des formes d'auto-organisation qui confortent la spécificité du périurbain dont elles sont issues.

Les réseaux et formes d'entraide que nous avons pu repérer permettent finalement de diminuer la vulnérabilité des habitants périurbains, que ce soit face aux aléas des déplacements ou face à de nouvelles contraintes, économiques et idéologiques. Ils donnent lieu à des formes d'organisation plus ou moins spontanées et pérennes. Cependant, pour s'actualiser, la ressource que constituent les relations sociales doit être constamment ajustée à la mobilité. Elle ne fonctionne pas totalement comme une ressource «territorialisée » se caractérisant par « des modes d'expression singuliers dans un lieu » [32], mais se spécifie en fonction d'une spatialité - l'itinéraire -, d'une temporalité - les agendas du déplacement -, et d'une technologie - les outils de communication -, la dimension fonctionnelle du type de transport différenciant notablement la forme des solidarités activées.

L'idée de ressource suppose par ailleurs une construction : les relations sociales ne sont pas assimilables à un vivier dans lequel l'habitant périurbain viendrait puiser au gré de ses besoins. En tant que ressources virtuelles [33], elles n'existent qu'à travers un processus d'activation, lui-même fonction de l'intentionnalité des acteurs. « La ressource est donc relative au contexte spatial et temporel qui l'a vu naître » [34].

\section{Conclusion}

Cette analyse des sociabilités dans le mouvement nous a permis d'appréhender les ajustements sociaux opérés en regard de la mobilité considérée en tant qu'action, à travers son organisation, son appropriation et ses formes. Ces ajustements sont donc situés dans leurs rapports à des modes et des réseaux de transports comme à des spatialités. Il en ressort un régime de mobilité, fortement spécifié par le contexte périurbain. Comment se caractérise-t-il ?

Les situations de mobilité périurbaine que nous avons étudiées se caractérisent d'abord par une temporalité particulière. La durée du déplacement apparaît suffisamment longue pour pouvoir générer des possibilités d'activités ou de rencontres propres, qui ne sont ni celles du voyage à longue distance, ni celles séparant deux arrêts de métro ou de tram. Cette durée, du moins hors région parisienne, donne lieu à des types de transport offrant un confort relatif ou des aménagements matériels, qui eux aussi contribuent à la réalisation du potentiel d'activités. $\mathrm{Si}$, par exemple, la durée d'un parcours en tramway au sein de l'agglomération peut être importante pour certains, l'aménagement intérieur, la fréquence des arrêts, la proportion de places assises, ne permettront pas de s'approprier cet espace-temps de la même manière que dans un des modes de transport périurbain que nous avons observés. La mobilité périurbaine, lorsqu'elle implique un recours aux transports collectifs ou au covoiturage, offre donc des conditions d'habitabilité propices à l'appropriation des territoires de la mobilité que l'on retrouverait probablement dans les déplacements interurbains, eux-mêmes en croissance. Dans ce contexte, la répétition routinière des déplacements renforce les mécanismes d'appropriation et les sociabilités observées. Elles créent les familiarités et les habitus par lesquels se constituent des codes d'usage, des relations stables ou des systèmes organisationnels pérennes. $\mathrm{Si}$, confrontée à la temporalité du quotidien, la durée du trajet donne lieu à des interactions sociales souvent faibles et toujours éphémères, ce sont les routines engagées dans la mobilité qui en permettent une territorialisation.

Nous avons également révélé une sociabilité conditionnée par l'espace-temps de la mobilité. Les usages, activités ou codes sociaux montrent la diversité et l'individualisation des modes d'appropriation. Mais cette diversité n'est nullement corrélée à du repli sur soi et à du côtoiement sans interaction. Il apparaît plutôt des formes de gestion très fines des interactions, manifestant une grande flexibilité et de l'adaptabilité au souhait d'interactions des personnes présentes. Il y a beaucoup d'empathie dans le fait d'identifier et d'accepter que certaines personnes puissent ne pas avoir envie de discuter. Plutôt que de l'indifférence, cette sensibilité révèle des formes de tolérance, ou la reconnaissance d'états d'esprit que l'on peut soi-même éprouver et qu'on sait liés à la condition périurbaine. Se prêtant parfois à des échanges d'une grande intimité dans le huis clos d'une automobile, parfois, technologie aidant, à une totale absence vis-à-vis des co-navetteurs présents, la mobilité forme un espace à la sociabilité peu normée. En regard des relations codées d'un voisinage résidentiel ou d'un milieu professionnel, il n'existe pas de comportement obligé, mais une latitude analogue à celle que permet un espace public en milieu urbain. La gestion des interactions montre également la reconnaissance de cette temporalité particulière du «sas ». La disponibilité de chacun est fonction du moment dans lequel il se situe dans le passage entre les lieux fixes de ses activités : encore dans le travail, dans sa sphère domestique, ou dans l'habitacle avec ses voisins pour un instant d'échanges. L'échange n'est donc pas seulement lié à une co-présence, mais aussi à une synchronisation dans ces différentes phases mentales.

Cette sociabilité et les activités pratiquées dessinent ainsi ce que l'on pourrait appeler un habiter propre à la mobilité. 
Mais ce régime de mobilité a également pour particularité l'interconnexion étroite entre l'habiter de l'ancrage et l'habiter mobile. Nous avons vu cette progression dans les activités, avec un changement d'état d'esprit, d'activité, ou d'ouverture aux autres et à l'environnement qui témoigne que le moment du déplacement ne fait pas rupture mais crée une continuité territoriale. Les réseaux sociaux, de travail, résidentiels, amicaux, constituent par ailleurs une ressource essentielle pour assurer la continuité dans la réalisation de la mobilité. À la solidarité spontanée et informelle que suscite une immobilité contrainte, s'ajoutent des routines dans les arrangements qui conduisent à structurer des collectifs d'entraide et d'organisation. Les liens tissés à l'occasion de la mobilité percolent par ailleurs dans les espaces d'habitat. Ils participent à donner sens et valeur à la proximité spatiale et ainsi à conforter des collectifs inscrits dans l'espace résidentiel de voisinage. Cette interconnexion structure d'une certaine manière un «système de mobilité localisé ». À l'image du Système productif localisé (SPL), où la force des liens locaux structure et valorise la production, les réseaux sociaux issus de la mobilité participent à la production de réseaux sociaux territorialisés. Outre l'objet commun de la mobilité, ils sont également portés par des valeurs idéelles : la conviction écologique et la confiance localisée reposent sur le partage d'une même identité locale.

La spatialité de ce système est toutefois particulière. Elle n'est pas fondée sur une logique de distance géographique, qui organiserait la ressource sur une logique de proximité à un lieu fixe d'activités (travail ou résidence), mais sur une logique réticulaire de partage d'un même segment de réseau et plus généralement d'une même condition mobilitaire relative à l'habiter périurbain. Le réseau se fait entre personnes se déplaçant entre les mêmes nœuds d'intermodalité. Les lieux de destination ou de départ collectifs ne sont pas en effet les lieux de résidence ou de travail, mais ceux ouvrant vers un nouveau type de réseau permettant de desservir ce dernier maillon : tramway en agglomération, stop ou marche dans l'espace périurbain, vélo pour l'un ou l'autre.

$\mathrm{Au}$ bout du compte, ce régime de mobilité sous-tend une production d'identités collectives de mouvement. Il s'agit là d'identités qui, au-delà de la pluralité et de la labilité de leurs formes, se fondent sur le partage des mêmes conditions matérielles et idéelles de mobilité ainsi que sur une synchronie dans les agendas d'activités et dans la gestion de la transition territoriale (le « sas »). Particulièrement saillantes dans le cas du périurbain (mais on peut imaginer qu'elles structurent d'autres modes d'habiter), ces identités liées au mouvement entrent en résonance, et d'une certaine manière se nourrissent, des autres composantes de l'identité socio-spatiale (résidentielles, professionnelles, récréatives, etc.), et en retour, participent de manière centrale et déterminante à la fabrique des identités de déplacement (au sens de Ramadier) et plus largement encore des modes d'habiter. Ainsi, l'identité de mouvement participe de l'identité résidentielle périurbaine, elles se co-construisent, s'intériorisent et s'identifient dans les interactions mobilisées dans la mobilité. Dans le mouvement s'élaborent les ferments d'une identification collective au territoire qui articule la résidence comme lieu situé au sein d'un territoire commun et le déplacement comme enjeu partagé objectivé dans la mobilité et lié à cette condition résidentielle.

\section{Bibliographie}

1. Lanéelle $X$ (2005) Réseau social, réseau ferroviaire. In : Montulet B, Hubert M, Jemelin C, Schmitz S (dir.) Mobilités et temporalités. Publications Facultés universitaires Saint-Louis, collection « Travaux et recherches », pp. 197-206.

2. Frétigny J-B (2006) L'espace du train en Italie dans une perspective de géographie culturelle. Mémoire de Master Géographie, Université Paris 1.

3. Pinson D, Thomann S (2001) La maison en ses territoires, de la villa à la ville diffuse. L'Harmattan, $191 \mathrm{p}$.

4. David O (2013) Les équations temporelles et spatiales des familles périurbaines. EspacesTemps.net, 22/05/2013 [En ligne] url : http://www.espacestemps.net/articles/les-equations-temporelleset-spatiales-des-familles-periurbaines/

5. André-Poyaud I, Chardonnel S, Charleux L, Tabaka K (2008) La mobilité au cœur des emplois du temps des citadins. In: Chalas Y, Paulhiac F (eds.) La mobilité qui fait la ville. Éditions du Certu, pp 67-95.

6. Chalas Y, Dubois-Taine G (1997) La ville émergente. Éditions de l'Aube, collection « Monde en cours », 285 p.

7. Hervouët V (2007) La mobilité du quotidien dans les espaces périurbains, une grande diversité de modèles de déplacements. Norois $205:$ 37-52.

8. Cailly L (2008) Existe-t-il un mode d'habiter spécifiquement périurbain? EspacesTemps.net, 30/5/2008, [En ligne] http://test. espacestemps.net/articles/existe-t-il-un-mode-drsquohabiterspecifiquement-periurbain/

9. Motte B (2007) Les populations périurbaines face à l'automobile en grande couronne francilienne. Norois 205 : 53-66.

10. Lévy J (2001) Préface. In: Pinson D, Thomann S (dir.) La maison en ses territoires, de la villa à la ville diffuse. L'Harmattan.

11. Cailly L (2010) La question périurbaine revisitée. In: Cailly L, Vanier L. (dir.) France, une géographie urbaine. Armand Colin, pp. 214-234.

12. Feildel B, Bailleul H, Laffont G-H (2014) Le périurbain à l'épreuve des imaginaires de la mobilité. De possibles ressorts pour la mise en durabilité des espaces périurbains. Recherche Transports Sécurité (à paraître).

13. Dodier R (2012) Habiter les espaces périurbains. PUR, 220 p.

14. Guilbot O (1979) Vers une analyse stratégique de la sociabilité. CNRS, Archives de l'OCS, vol. 1, pp. 7-31.

15. Gurvitch G (1963) La vocation de la sociologie. PUF, t.1, pp. 118-247.

16. Rivière C-A (2004) La spécificité française de la construction sociologique du concept de sociabilité. Réseaux 123 : 207-231.

17. Simmel G (1999) Sociologie, étude sur les formes de la socialisation. PUF, $776 \mathrm{p}$.

18. Brubaker R (2001) Au-delà de l'identité. Actes de la recherche en sciences sociales 139(4) : 66-85. 
19. Ramadier T, Lannoy P, Depeau S et al. (2009) Vers l'hypothèse d'une identité de déplacement : congruence entre espace social, cognitif et géographique. In: Grandjean $\mathrm{P}$, (dir.) Construction identitaire et espace. L'Harmattan, pp. 75-94.

20. TerrHab-mobile (2013) Lorsque la mobilité territorialise. EspacesTemps.net, 13/05/2013, [En ligne] http://www. espacestemps.net/articles/lorsque-la-mobilite-territorialise/

21. Piolle X (1991) Proximité géographique et lien social, de nouvelles formes de territorialité ? L'espace géographique 4 : 349-358.

22. Miaux S (2008) Comment la façon d'envisager la marche conditionne la perception de l'environnement urbain et le choix des itinéraires piétonniers - L'expérience de la marche dans deux quartiers de Montréal, Recherche Tensports Sécurité 101 : $327-351$.

23. Lévy E (2001) Saisir l'accessibilité : les trajets-voyageurs à la gare du Nord. In : Grosjean M, Thibaud J-P (éds.) L'espace urbain en méthodes, Parenthèses, pp. 47-62.

24. Audas N, Martouzet D (2008) Saisir l'affectif urbain. Proposition originale par la cartographie de réactivation des discours, communication au colloque Penser la ville - approches comparatives, Khenchela, Algérie [En ligne] $\mathrm{http}: / /$ halshs.archives-ouvertes.fr/docs/00/38/05/53/PDF/Microsoft Word-Saisirlaffectifurbain.pdf

25. Sajous P (2008) Enquête dans un quartier de la ville d'Albi sur les perceptions et les attentes des piétons en matière d'éclairage. Recherche Transports sécurité 101:353-368.

26. Lussault M (2009) De la lutte des classes à la lutte des places. Grasset, $254 \mathrm{p}$.

27. Dodier R (2007) Temporalités périurbaines : des navettes pendulaires à la fluidité et au conflit. Espace, populations, sociétés $2 / 3: 305-316$.

28. Lévy J, Lussault M (dir.) (2003) Dictionnaire de la géographie et de l'espace des sociétés, Belin.

29. Rougé L (2011) Mobilités et modes de vie dans les espaces faiblement denses du périurbain toulousain : entre recomposition des clivages socio-spatiaux et formes d'autonomisation. In : Dumont M., Hellier E (dir.) Les nouvelles périphéries urbaines, PUR, $218 \mathrm{p}$.

30. Rémy J (2004) Culture de la mobilité et nouvelles formes de territorialité. In: Vodoz L, Pfister GB, Jemelin C (eds) Les territoires de la mobilité, l'aire du temps. Presses polytechniques et universitaires romandes, pp 13-42.

31. Dodier (2009) Individus et groupes sociaux dans l'espace, apports à partir de l'exemple des espaces périurbains. Habilitation à diriger les recherches de géographie, Université du Maine.

32. Louargant S, Bensahel L (2007) Une approche de la notion de ressources territorialisées par la problématique de genre. In: Gumuchian H, Pecqueur B (éds.) La ressource territoriale, Economica, pp. 191-208.
33. Pecqueur B (2006) Le tournant territorial de l'économie globale. Espace et Société 124-125: 17-32.

34. Lamara H (2009) Les deux piliers de la construction territoriale : coordination des acteurs et ressources territoriales. Développement durable et territoires, 7/7/2009, [En ligne] http://developpementdurable.revues.org/8208 ; DOI : 10.4000/developpementdurable. 8208

\section{Liste des enquêtés}

- Isabelle, 40 ans, employée, voiture personnelle solo.

- Sylvain, 24 ans, employé, bus.

- Aurélie, 26 ans, étudiante, bus.

- Nicole, 50 ans, employée, bus.

- Claudine, 57 ans, profession intermédiaire, voiture personnelle solo et avec son mari.

- Annick, 60 ans, employée, voiture personnelle solo.

- Marie, 52 ans, cadre, voiture personnelle solo et covoiturage

- Claude, 49 ans, profession intellectuelle supérieure, voiture personnelle et TER.

- Sébastien, 26 ans, employé, voiture personnelle solo.

- Caroline, 27 ans, cadre, voiture personnelle solo.

- Hervé, 55 ans, employé, voiture personnelle solo ou bus, et TER.

- Simon, 39 ans, profession intellectuelle supérieure, covoiturage.

- Olivier, 36 ans, cadre de la fonction publique, voiture personnelle solo et vélo.

- Fabrice, 52 ans, profession intermédiaire, voiture personnelle solo ou en covoiturage.

- Jean-Pierre, 50 ans, profession intellectuelle supérieure, covoiturage.

- Laurence, profession libérale, voiture personnelle solo ou en covoiturage.

- Antoine, 38 ans, cadre de la fonction publique, voiture personnelle solo et bus.

- Anne, 41 ans, cadre de la fonction publique, voiture personnelle solo ou en covoiturage et bus. 